\title{
PREVENTABLE DELAYS IN THE TREATMENT OF INTRAOCULAR MELANOMA IN THE UK
}

\author{
ROGER HOLDEN and BERTIL E. DAMATO \\ Liverpool
}

\begin{abstract}
SUMMARY
Intraocular melanoma should be treated urgently to improve the chances of survival and of conserving the eye and vision. The aims of this study were to determine the mode of presentation of intraocular melanoma in the United Kingdom and to identify any preventable causes of delay in treatment. A total of $\mathbf{1 1 2}$ patients were included and questioned about their initial symptoms, the type of practitioner consulted and their subsequent management. Thirty per cent of patients were asymptomatic and detected on routine ophthalmoscopy. Symptomatic patients presented with blurred vision (52\%), photopsia (26\%), visual field loss (9\%) and miscellaneous symptoms $(13 \%)$. Patients presented to an optometrist in 66 cases $(59 \%)$, to a general practitioner in 30 cases $(27 \%)$ and to an ophthalmologist in 16 cases $(14 \%)$. The tumour was misdiagnosed in $12 \%$ of patients presenting to an optometrist and in $27 \%$ of cases presenting to a GP. The period from the time of onset of symptoms or detection of an abnormality to the time when the patient was referred to the Ocular Oncology Service averaged 6 months (range 0-36 months). Patients waited an average of $\mathbf{4}$ months if referred urgently as compared with 7.7 months if referred non-urgently (Mann-Whitney $U$-test, $p=0.0003$ ). Patients were likely to be treated sooner if they were referred directly to the ophthalmologist rather than via the GP (7.8 months vs 4.8 months; Mann-Whitney $U$-test, $p=0.02$ ).
\end{abstract}

Intraocular melanoma is a malignant neoplasm of the eye which affects about 6 persons per million per year. ${ }^{1,2}$ About $50 \%$ of patients die of metastases within 10 years of enucleation, the risk of metastases being related to factors such as tumour size, cell type and age at treatment. ${ }^{3,4}$ If the patient is seen early enough it may be possible to conserve the eye and vision by a variety of methods which include plaque

From: Ocular Oncology Service, St Paul's Eye Unit, Royal Liverpool University Hospital, Prescot Street, Liverpool, UK.

Correspondence to: Roger Holden, FRCOphth, Derbyshire Royal Infirmary, London Road, Derby DE1 8QY, UK. Fax: ++44 (01332) 254825. radiotherapy, proton beam radiotherapy, laser photocoagulation, trans-scleral local resection and trans-vitreal 'endoresection'., therefore diminishes the chances of both survival and retention of a useful eye. ${ }^{7,8}$

\section{MATERIALS AND METHODS}

The sample included all British patients treated for intraocular melanoma at the Ocular Oncology Service (OOS) at Liverpool in 1993. Patients were interviewed by the same investigator (R.H.) about their symptoms and the health care personnel to which they presented. The questionnaires were conducted either in the hospital clinic or over the telephone. Information was obtained on: (i) age and gender, (ii) nature and duration of symptoms, (iii) date of detection of abnormality by any practitioner, (iv) type of practitioner detecting abnormality, (v) initial diagnosis and management, (vi) subsequent management, (vii) visual acuity, (viii) eye laterality, (ix) anteroposterior location of tumour, (x) tumour dimensions and (xi) type of treatment. Ethics committee approval was obtained for the study.

Due to the non-Gaussian distribution of the data, non-parametric tests were used: (i) the MannWhitney $U$-test for continuous data, (ii) the chisquared test for categorical data and (iii) Spearman's rank correlation test. A probability of $\leqslant 0.05$ was taken as significant.

\section{RESULTS}

Patients

Three hundred and thirteen patients were referred to the OOS with a suspected tumour during 1993. Of these, 141 patients were treated for intraocular melanoma, 120 being from the UK and 21 from overseas. Four patients could not be contacted and another 4 had previously received treatment at another centre, leaving 112 British patients who could be included in the study. These comprised 63 
women and 49 men with a mean age of 61 years (range 20-87 years).

\section{Tumours}

All melanomas were unilateral, 57 (50.8\%) being located in the left eye and $55(49.2 \%)$ in the right eye. The tumours were situated in the choroid (90, $80 \%)$, ciliary body $(14,13 \%)$ and iris $(8,7 \%)$. In 101 measurable tumours, the mean largest basal diameter was $12.2 \mathrm{~mm}$ (range $5-21 \mathrm{~mm}$ ) and the mean thickness was $5.6 \mathrm{~mm}$ (range $2-12 \mathrm{~mm}$ ).

\section{Symptoms}

Seventy-eight patients $(70 \%)$ presented with symptoms, the remaining $34(30 \%)$ being detected on routine examination which was performed either by optometrists (31) or by ophthalmologists screening for diabetic retinopathy (3). No asymptomatic patients were detected by the general practitioner. The main symptoms were: (i) blurred vision (41, $52 \%$ ), (ii) photopsia $(20,26 \%$ ), (iii) visual field loss (7, 9\%) and (iv) miscellaneous symptoms such as ocular pain $(10,13 \%)$. The presence of symptoms was related to tumour thickness (Mann-Whitney $U$ test, $p=0.02$ ) and largest tumour diameter (MannWhitney $U$-test, $p=0.001$ ), but not to posterior extension (chi-squared test, $p=0.54$ ).

\section{Visual Acuity}

Snellen visual acuity in the affected eye was $6 / 12$ or better in 66 patients $(59 \%), 6 / 18-6 / 60$ in 32 patients $(28.5 \%)$ and worse than $6 / 60$ in 14 patients $(12.5 \%)$.

\section{Presentation}

Patients initially presented to an optometrist in 66 cases $(59 \%)$, to a general practitioner in 30 cases $(27 \%)$ and to an ophthalmologist in 16 cases (14\%). The tumour was misdiagnosed or missed in 8 patients presenting to an optometrist $(12 \%)$ and in 8 patients presenting to the general practitioner $(27 \%)$. Four melanomas were missed or misdiagnosed by the ophthalmologists because they were diagnosed as naevi or because of vitreous haemorrhage.

\section{Delay}

In the symptomatic patients, the time interval between onset of symptoms and initial presentation was 2.2 months (range 0-24 months). The period from the time of onset of symptoms or detection of abnormality to the time when the patient was referred to the OOS averaged 6 months (range 0-36 months). Thirty-seven cases were referred urgently to an ophthalmologist, 29 of these by optometrists $(78 \%)$ and $8(22 \%)$ by general practitioners. Patients referred urgently tended to wait 4 months to be seen by an ophthalmologist as compared with 7.7 months in those patients referred non-urgently. This difference in waiting times was statistically significant (Mann-Whitney $U$-test, $p=0.0003$ ).

Patients attending three or more practitioners waited an average of 7.8 months for treatment, as compared with 4.8 months for patients attending fewer practitioners (Mann-Whitney $U$-test, $p=0.02$ ). This result indicated that patients were treated more quickly if their optometrist referred them directly to the ophthalmologist instead of via the general practitioner.

Of the 20 patients who were initially misdiagnosed, 8 sought an alternative opinion within 2 weeks, but another 6 experienced a delay of more than 1 year. There was no signfiicant correlation between length of delay and tumour size as indicated by tumour thickness and basal diameter (Spearman's rank correlation test: $r=0.004, p=0.7$ and $r=0.0002$, $p=0.98$ respectively).

The mean time interval between date of referral to the OOS and the first visit there was 1.8 weeks (range $0-6$ weeks). The time between the first visit to the OOS and treatment was less than 2 days in 68 cases $(61 \%), 2-14$ days in 32 cases $(28 \%)$ and more than 2 weeks in 12 cases $(11 \%)$. These delays were mainly due to the need for patients to consider various therapeutic options or to make domestic arrangements for their stay in hospital.

\section{Treatment}

The 112 patients in the study were treated by proton beam radiotherapy $(37 \%)$, enucleation $(27 \%)$, transscleral local resection (18\%), plaque radiotherapy (14\%) and trans-vitreal local 'endoresection' (4\%).

\section{DISCUSSION}

It is likely that the results of this study reflect the current situation throughout the United Kingdom because of the nation-wide source of referrals. The tumour distribution within the eye is in keeping with previous studies, indicating that the sample is representative. Although the diagnosis of intraocular melanoma was not proven histologically in the many cases treated by radiotherapy, it is known that with current methods of examination the rate of misdiagnosis in specialist centres is less than $1 \%{ }^{9}$

The most important finding of this study is that the mean delay between first presentation and treatment was 6 months, and exceeded 1 year in $14 \%$ of cases. The main reason for such delay was failure of the general practitioner or optometrist to ensure that the patient was seen urgently by an ophthalmologist. In most patients, non-urgent referral occurred because the cause of the visual symptoms had not been established. Almost $20 \%$ of patients were misdiagnosed or wrongly discharged. Delay was longer when 
patients were referred by the optometrist to the ophthalmologist via the general practitioner than when they were referred directly. Some patients contributed to the delay, suggesting they had not been made aware of the gravity of the suspected diagnosis. That no correlation between delay in referral and tumour size was found is not surprising in view of the many other confounding factors.

This study suggests that the generally accepted protocols for examining the eye in the community are inadequate. Optometrists and general pracitioners could benefit from further education about the relevance of an afferent pupillary defect and the importance of dilating the pupil before performing ophthalmoscopy (preferably with an indirect ophthalmoscope). The results also imply that patients with suspected intraocular melanoma should be informed of the suspected diagnosis so that they could help minimise delays in treatment. Finally this study suggests that treatment is delayed significantly when the patient is referred by an optometrist via the general practitioner. Measures should be taken to ensure patients are seen urgently by an ophthalmologist.

In conclusion there is scope for reviewing guidelines for the management of patients with visual symptoms, with regard to both method of examination and referral to the ophthalmologist.

Key words: Delay, Intraocular, Melanoma, Treatment.

\section{REFERENCES}

1. Scotto J, Fraumeni JF, Lee JAH. Melanomas of the eye and other non-cutaneous sites: epidemiological aspects. J Natl Cancer Inst 1976;56:489-91.

2. Egan KM, Seddon JM, Glynn RJ, Gragoudas ES, Albert DM. Epidemiological aspects of uveal melanoma. Surv Ophthalmol 1988;32:239-51.

3. Raivio I. Uveal melanoma in Finland: an epidemiological, clinical, histological and prognostic study. Acta Ophthalmol Suppl 1977;133:3-64.

4. Jensen OA. Malignant melanomas of the human uvea: 25 year follow-up of cases in Denmark, 1942-1952. Acta Ophthalmol (Copenh) 1982;60:161-82.

5. Shields JA. Management of uveal melanoma: a continuing dilemma. Cancer 1993;72:2067-8.

6. Damato BE. An approach to the management of patients with uveal melanoma. Eye 1993;7:388-97.

7. Seddon JM, Gragoudas ES, Egan KM, et al. Relative survival rates after alternative therapies for uveal melanoma. Ophthalmology 1990;97:769-77.

8. Zimmerman LE, McLean IW. An evaluation of enucleation in the management of uveal melanomas. Am J Ophthalmol 1979;87:741-60.

9. Char DH, Stone RD, Irvine AR, Crawford JB, Hilton GF, Lonn LI, Schwartz A. Diagnostic modalities in choroidal melanoma. Am J Ophthalmol 1980;89:223-30. 